\title{
ON RELAXATION OF STATE-CONSTRAINED OPTIMAL CONTROL PROBLEM IN COEFFICIENTS FOR BIHARMONIC EQUATION
}

\author{
P. I. Kogut*, L. V. Voloshko** \\ * Department of Differential Equations, Dnipropetrovsk National University, \\ Gagarin av., 72, Dnipropetrovsk, 49010, e-mail: p.kogut@i.ua \\ ** Department of Applies Mathematics, Dnipropetrovsk National University, \\ Gagarin av., 72, Dnipropetrovsk, 49010.e-mail: lyubov@voloshko.com
}

We study a Dirichlet optimal control problem for biharmonic equation with control and state constraints. The coefficient of the biharmonic operator, the weight $u$, we take as a control in $L^{1}(\Omega)$. We discuss the relaxation approach and show that some optimal solutions to the original problem can be attained in the limit by optimal solutions of some extremal problem for variational inequality with a special penalized cost functional.

Key words: biharmonic problem, optimal control, control in coefficients, relaxation, existence result.

\section{Introduction}

The aim of this article is to analyze an optimal control problem for a linear PDE with mixed boundary conditions where the coefficient of bilaplacian operator we take as a control. Since an important matter for applications is to obtain a solution to a given boundary problem with desired properties, it leads to the reasonable questions: can we define an appropriate coefficient of bilaplacian operator to minimize the discrepancy between a given displacement $y_{d}$ and an expected solution to such problem.

The characteristic feature of OCP is the fact that the solutions of the boundary value problem should be restricted by some pointwise constraints in $L^{2}$-space. As for the class of admissible controls, we consider it as a nonempty subset of $L^{1}(\Omega)$ with an empty topological interior. Such choice is motivated by needs of having good properties of solutions to the corresponding boundary value problem.

The outline of the paper is the following. In Section 2 we report some preliminaries and notation we need in the sequel. In Sections 3 we give the precise statement of the state constrained optimal control problem and describe the main assumptions on the initial data and control functions. In Section 4 we provide the results concerning solvability of the original problem with control and state constraints. We show that this problem admits at least one solution if and only

(C) P. I. Kogut, L. V. Voloshko, 2015 
if this problem is regular, that is, the corresponding set of admissible solutions is nonempty. In Section 5 we show that the pointwise state constraints can be relaxed and discuss to the approximation of OCPs, called the "variational inequality method". Following this approach, we weaken the requirements on admissible solutions to the original OCP. As a result, we propose another interpretation of relaxation for the state-constrained OCPs and give the statement of this relaxation in the form of some optimal control problem for variational inequality with a special penalized cost functional. We show that he penalized optimal control problem for indicated variational inequality is always regular and solvable. In conclusion, we show that some optimal solutions to the original problem can be attained in the limit by optimal solutions of the penalized problem. However, it is unknown whether the entire set of the optimal solutions can be attained in such way.

\section{Definitions and Basic Properties}

Let $\Omega$ be a bounded open connected subset of $\mathbb{R}^{N}(N \geq 2)$. We assume that the boundary $\partial \Omega$ is Lipschitzian so that the unit outward normal $\nu=\nu(x)$ is well-defined for a.e. $x \in \partial \Omega$, where a.e. means here with respect to the $(N-1)$ dimensional Hausdorff measure. We also assume that the boundary $\partial \Omega$ consists of two disjoint parts $\partial \Omega=\Gamma_{D} \cup \Gamma_{S}$, where the sets $\Gamma_{D}$ and $\Gamma_{S}$ have positive $(N-1)$-dimensional measures, and $\Gamma_{S}$ is now $C^{2}$.

By $W^{2,2}(\Omega)$ we denote the Sobolev space as the subspace of $L^{2}(\Omega)$ of functions $y$ having generalized derivatives $D^{k} y$ up to order $k=2$ in $L^{2}(\Omega)$. We note that due to the interpolation theory, see [1, Theorem 4.14], $W^{2,2}(\Omega)$ is a Banach space with respect to the norm

$$
\|y\|_{W^{2,2}(\Omega)}=\left(\|y\|_{L^{2}(\Omega)}^{2}+\left\|D^{2} y\right\|_{L^{2}(\Omega)}^{2}\right)^{1 / 2}=\left(\int_{\Omega}\left(|y|^{2}+\left|D^{2} y\right|^{2}\right) d x\right)^{1 / 2}
$$

where

$$
D^{2} y \cdot D^{2} v=\left(\sum_{i_{1}, i_{2}=1}^{N} \frac{\partial^{2} y}{\partial x_{i_{1}} \partial x_{i_{2}}} \frac{\partial^{2} v}{\partial x_{i_{1}} \partial x_{i_{2}}}\right)^{1 / 2} \quad, \quad \text { and } \quad\left|D^{2} y\right|=\left(D^{2} y \cdot D^{2} y\right)^{1 / 2} \text {. }
$$

For any $y \in C^{1}(\bar{\Omega})$ we define the traces

$$
\gamma_{0}(y)=\left.y\right|_{\partial \Omega}, \text { and } \gamma_{1}(y)=\left.\frac{\partial y}{\partial \nu}\right|_{\partial \Omega} .
$$

By [14, Theorem 8.3], these linear operators can be extended continuously to the whole of space $W^{2,2}(\Omega)$. We set

$$
W^{3 / 2,2}(\partial \Omega):=\gamma_{0}\left[W^{2,2}(\Omega)\right], \quad W^{1 / 2,2}(\partial \Omega):=\gamma_{1}\left[W^{2,2}(\Omega)\right]
$$


as closed subspaces of $W^{1,2}(\partial \Omega)$ and $L^{2}(\partial \Omega)$, respectively. Moreover, the injections

$$
W^{3 / 2,2}(\partial \Omega) \hookrightarrow W^{1,2}(\partial \Omega) \quad \text { and } \quad W^{1 / 2,2}(\partial \Omega) \hookrightarrow L^{2}(\partial \Omega)
$$

are compact.

Let $C_{0}^{\infty}\left(\mathbb{R}^{N} ; \Gamma_{D}\right)=\left\{\varphi \in C_{0}^{\infty}\left(\mathbb{R}^{N}\right): \varphi=0\right.$ and $\frac{\partial \varphi}{\partial \nu}=0$ on $\left.\Gamma_{D}\right\}$. We define the Banach space $H_{0}^{2}\left(\Omega ; \Gamma_{D}\right):=W_{0}^{2,2}\left(\Omega ; \Gamma_{D}\right)$ as the closure of $C_{0}^{\infty}\left(\mathbb{R}^{N} ; \Gamma_{D}\right)$ with respect to the norm $\|y\|_{W^{2,2}(\Omega)}$. Let $H^{-2}\left(\Omega ; \Gamma_{D}\right)$ be the dual space to $H_{0}^{2}\left(\Omega ; \Gamma_{D}\right)$. We also define the space $H_{0}^{1}(\Omega)$ as the closure of $C_{0}^{\infty}(\Omega)$ with respect to the norm $\|y\|_{H_{0}^{1}(\Omega)}=\left(\int_{\Omega}\|\nabla y\|_{\mathbb{R}^{N}}^{2} d x\right)^{1 / 2}$.

Throughout this paper, we use the notation $\mathbb{W}_{2}(\Omega):=H_{0}^{2}\left(\Omega ; \Gamma_{D}\right) \cap H_{0}^{1}(\Omega)$. Let us notice that $\mathbb{W}_{2}(\Omega)$ equipped with the norm

$$
\|y\|_{2, \Delta}:=\|\Delta y\|_{L^{2}(\Omega)}=\left(\int_{\Omega}|\Delta y|^{2} d x\right)^{1 / 2}=\left(\int_{\Omega}\left|\sum_{i=1}^{N} \frac{\partial^{2} y}{\partial x_{i}^{2}}\right|^{2} d x\right)^{1 / 2}
$$

is a uniformly convex Banach space [4]. Moreover, the norm $\|\cdot\|_{2, \Delta}$ is equivalent on $\mathbb{W}_{2}(\Omega)$ to the usual norm of $W^{2,2}(\Omega)$. Indeed, since the Laplace operator $-\Delta$ acts from $\mathbb{W}_{2}(\Omega)$ in $L^{2}(\Omega)$ and the Dirichlet boundary value problem

$$
\Delta y=f \text { in } \Omega, \quad y=0 \text { on } \partial \Omega
$$

is uniquely solvable in $\mathbb{W}_{2}(\Omega)$ for all $f \in L^{2}(\Omega)$, it follows that the inverse operator $T:=(-\Delta)^{-1}: L^{2}(\Omega) \rightarrow W^{2,2}(\Omega) \cap H_{0}^{1}(\Omega)$ is well defined and satisfies the following elliptic regularity estimate [9]

$$
\|T f\|_{W^{2,2}(\Omega)} \leq C_{2}\|f\|_{L^{2}(\Omega)} .
$$

This allows us to conclude the following. If $f \in L^{2}(\Omega)$ and $y \in H_{0}^{1}(\Omega)$ are such that $\frac{\partial y}{\partial \nu}=0$ on $\Gamma_{D}$ and $y$ is a solution of $(2.3)$, then $-\Delta y \in L^{2}(\Omega), y=0$ on the boundary $\partial \Omega$, and, therefore, $y \in \mathbb{W}_{2}(\Omega)$. Hence,

$$
\|y\|_{W^{2,2}(\Omega)}=\|T(-\Delta y)\|_{W^{2,2}(\Omega)} \leq C_{2}\|\Delta y\|_{L^{2}(\Omega)}=C_{2}\|y\|_{2, \Delta},
$$

for a suitable positive constant $C_{2}$ independent of $f$. On the other hand, it is easy to remark that

$$
\|y\|_{2, \Delta} \leq\|y\|_{W^{2,2}(\Omega)} .
$$

Thus, by the Closed Graph Theorem, we can conclude that $\|\cdot\|_{2, \Delta}$ is equivalent to the norm induced by $W^{2,2}(\Omega)$ (for the details we refer to $[6,15]$ ).

By $B V(\Omega)$ we denote the space of all functions in $L^{1}(\Omega)$ for which the norm

$$
\begin{aligned}
\|f\|_{B V(\Omega)} & =\|f\|_{L^{1}(\Omega)}+\int_{\Omega}|D f|=\|f\|_{L^{1}(\Omega)} \\
& +\sup \left\{\int_{\Omega} f \operatorname{div} \varphi d x: \varphi \in C_{0}^{1}\left(\Omega ; \mathbb{R}^{N}\right),|\varphi(x)| \leq 1 \text { for } x \in \Omega\right\}
\end{aligned}
$$


is finite.

We recall that a sequence $\left\{f_{k}\right\}_{k=1}^{\infty}$ converges weakly-* to $f$ in $B V(\Omega)$ if and only if the two following conditions hold (see [10]): $f_{k} \rightarrow f$ strongly in $L^{1}(\Omega)$ and $D f_{k} \rightarrow D f$ weakly- ${ }^{*}$ in the space of Radon measures $\mathcal{M}(\Omega$, i.e.

$$
\lim _{k \rightarrow \infty} \int_{\Omega} \varphi D f_{k}=\int_{\Omega} \varphi D f \quad \forall \varphi \in C_{0}(\Omega) .
$$

It is well-known also the following compactness result for $B V$-spaces (Helly's selection theorem, see [2]).

Theorem 2.1. If $\left\{f_{k}\right\}_{k=1}^{\infty} \subset B V(\Omega)$ and $\sup _{k \in \mathbb{N}}\left\|f_{k}\right\|_{B V(\Omega)}<+\infty$, then there exists a subsequence of $\left\{f_{k}\right\}_{k=1}^{\infty}$ strongly converging in $L^{1}(\Omega)$ to some $f \in B V(\Omega)$ such that $D f_{k} \stackrel{*}{\rightarrow} D f$ weakly-* in the space of Radon measures $\mathcal{M}(\Omega)$. Moreover, if $\left\{f_{k}\right\}_{k=1}^{\infty} \subset B V(\Omega)$ strongly converges to some $f$ in $L^{1}(\Omega)$ and satisfies $\sup _{k \in \mathbb{N}} \int_{\Omega}\left|D f_{k}\right|<+\infty$, then

$$
\begin{aligned}
& \text { (i) } f \in B V(\Omega) \text { and } \int_{\Omega}|D f| \leq \liminf _{k \rightarrow \infty} \int_{\Omega}\left|D f_{k}\right| \text {; } \\
& \text { (ii) } f_{k} \stackrel{*}{\rightarrow} f \text { in } B V(\Omega) .
\end{aligned}
$$

\section{Setting of the Optimal Control Problem}

Let $\xi_{1}, \xi_{2}$ be fixed elements of $L^{\infty}(\Omega) \cap B V(\Omega)$ satisfying the conditions

$$
0<\alpha \leq \xi_{1}(x) \leq \xi_{2}(x) \text { a.e. in } \Omega,
$$

where $\alpha$ is a given positive value.

Let $f \in H^{-2}\left(\Omega ; \Gamma_{D}\right), y_{d} \in L^{2}(\Omega)$, and $\zeta^{\max } \in L^{2}(\partial \Omega)$ be given distributions. The optimal control problem, we consider in this paper, is to minimize the discrepancy between $y_{d}$ and the solutions of the following state-constrained boundary valued problem

$$
\begin{gathered}
\Delta^{2}(u(x), y)=f(x) \quad \text { in } \quad \Omega \\
y=\frac{\partial y}{\partial \nu}=0 \quad \text { on } \Gamma_{D}, \quad y=\Delta y=0 \quad \text { on } \Gamma_{S}, \\
0 \leq \frac{\partial y(s)}{\partial \nu} \leq \zeta^{\max }(s) \quad \text { a.e. on } \Gamma_{S}
\end{gathered}
$$

by choosing an appropriate weight function $u \in \mathfrak{A}_{a d}$ as control. Here,

$$
\Delta^{2}(u, y):=\Delta(u \Delta y)
$$

is the operator of fourth order called the biharmonic operator, and the class of admissible controls $\mathfrak{A}_{a d}$ we define as follows

$$
\mathfrak{A}_{a d}=\left\{u \in L^{1}(\Omega) \mid \xi_{1}(x) \leq u(x) \leq \xi_{2}(x) \text { a.e. in } \Omega\right\} .
$$


It is clear that $\mathfrak{A}_{a d}$ is a nonempty convex subset of $L^{1}(\Omega)$ with an empty topological interior.

More precisely, we are concerned with the following optimal control problem

$$
\text { Minimize }\left\{I(u, y)=\int_{\Omega}\left|y-y_{d}\right|^{2} d x+\int_{\Omega}|D u|\right\}
$$

subject to the constraints (3.2)-(3.5).

Definition 3.1. We say that an element $y \in \mathbb{W}_{2}(\Omega)$ is the weak solution (in the sense of Minty) to the boundary value problem (3.2)-(3.3), for a given admissible control $u \in \mathfrak{A}_{a d}$, if

$$
\int_{\Omega} u \Delta \varphi(\Delta \varphi-\Delta y) d x \geq\langle f, \varphi-y\rangle_{H^{-2}\left(\Omega ; \Gamma_{D}\right) ; H_{0}^{2}\left(\Omega ; \Gamma_{D}\right)}, \forall \varphi \in C_{0}^{\infty}\left(\Omega ; \Gamma_{D}\right) .
$$

Remark 3.1. Since the set $C_{0}^{\infty}\left(\mathbb{R}^{N} ; \Gamma_{D}\right)$ is dense in $\mathbb{W}_{2}(\Omega)$, it follows that the elements $\varphi \in \mathbb{W}_{p}(\Omega)$ can be considered for the test functions in (3.7). Therefore, taking $\varphi=y+t w$ with $w \in \mathbb{W}_{p}(\Omega)$ and $t>0$, we obtain

$$
\int_{\Omega} u(\Delta y+t \Delta w) \Delta w d x \geq\langle f, w\rangle_{H^{-2}\left(\Omega ; \Gamma_{D}\right) ; H_{0}^{2}\left(\Omega ; \Gamma_{D}\right)}, \quad \forall w \in \mathbb{W}_{2}(\Omega) .
$$

Passing to the limit as $t \rightarrow 0$, we get

$$
\int_{\Omega} u \Delta y \Delta w d x \geq\langle f, w\rangle_{H^{-2}\left(\Omega ; \Gamma_{D}\right) ; H_{0}^{2}\left(\Omega ; \Gamma_{D}\right)}, \quad \forall w \in \mathbb{W}_{2}(\Omega) .
$$

Hence,

$$
\int_{\Omega} u \Delta y \Delta w d x=\langle f, w\rangle_{H^{-2}\left(\Omega ; \Gamma_{D}\right) ; H_{0}^{2}\left(\Omega ; \Gamma_{D}\right)} .
$$

It is worth to note that having applied Green's formula twice to operator $\Delta(u \Delta y)$ tested by $v \in C_{0}^{\infty}\left(\Omega ; \Gamma_{D}\right)$, we arrive at the identity

$$
\begin{aligned}
\int_{\Omega} \Delta(u \Delta y) v d x & =-\int_{\Omega}(\nabla(u \Delta y), \nabla v)_{\mathbb{R}^{N}} d x+\int_{\partial \Omega} \frac{\partial}{\partial \nu}(u \Delta y) v d \mathcal{H}^{N-1} \\
& =\int_{\Omega} u \Delta y \Delta v d x-\int_{\Gamma_{D}} u \Delta y \frac{\partial v}{\partial \nu} d \mathcal{H}^{N-1}-\int_{\Gamma_{S}} u \Delta y \frac{\partial v}{\partial \nu} d \mathcal{H}^{N-1} \\
& =\int_{\Omega} u \Delta y \Delta v d x-\int_{\Gamma_{S}} u \Delta y \frac{\partial v}{\partial \nu} d \mathcal{H}^{N-1} \quad \forall v \in C_{0}^{\infty}\left(\Omega ; \Gamma_{D}\right) .
\end{aligned}
$$

Hence, if $y$ as an element of $\mathbb{W}_{2}(\Omega):=H_{0}^{2}\left(\Omega ; \Gamma_{D}\right) \cap H_{0}^{1}(\Omega)$ is the weak solution of the boundary value problem (3.2)-(3.3) in the sense of Definition 3.1, then relations (3.2)-(3.3) are fulfilled as follows (for the details, we refer to [17, Siction 2.4.4] and [8, Section 2.4.2])

$$
\left.\begin{array}{c}
\Delta^{2}(u, y)=f \quad \text { in }\left(C_{0}^{\infty}\left(\Omega ; \Gamma_{D}\right)\right)^{*}, \\
\gamma_{0}(y)=0 \text { in } W^{3 / 2,2}(\partial \Omega), \\
\gamma_{1}(y)=0 \text { in } W^{1 / 2,2}\left(\Gamma_{D}\right), \\
\gamma_{0}(\Delta y)=0 \text { in } W^{-1 / 2,2}\left(\Gamma_{S}\right):=\left(W^{1 / 2,2}\left(\Gamma_{S}\right)\right)^{*} .
\end{array}\right\}
$$


In particular, taking $w=y$ in (3.8), this yields the relation

$$
\int_{\Omega} u|\Delta y|^{2} d x=\langle f, y\rangle_{H^{-2}\left(\Omega ; \Gamma_{D}\right) ; H_{0}^{2}\left(\Omega ; \Gamma_{D}\right)}
$$

which is usually referred to as the energy equality. As a result, conditions (3.1), (3.5), and inequality (2.4) lead us to the following a priori estimate

$$
\|y\|_{2, \Delta}:=\left(\int_{\Omega}|\Delta y|^{2} d x\right)^{1 / 2} \leq\left(\alpha^{-1} C_{2}\|f\|_{H^{-2}\left(\Omega ; \Gamma_{D}\right)}\right) \quad \forall u \in \mathfrak{A}_{a d} .
$$

The existence of a unique weak solution to the boundary value problem (3.2)(3.3) in the sense of Definition 3.1 follows from the celebrated Lax-Milgram Theorem. Indeed, let us the operator $A(u, \cdot): \mathbb{W}_{p}(\Omega) \rightarrow\left(\mathbb{W}_{p}(\Omega)\right)^{*}$ as follows

$$
\langle A(u, y), w\rangle_{\left(\mathbb{W}_{2}(\Omega)\right)^{*} ; \mathbb{W}_{2}(\Omega)}:=\int_{\Omega} u \Delta y \Delta w d x d x .
$$

It is easy to see that $A(u, y)=\Delta_{p}^{2}(u, y)$ and $A(u, \cdot)$ satisfies all assumptions of Lax-Milgram Theorem (for the details we refer to [13,17]). Hence, the variational problem

For a given $u \in \mathfrak{A}_{a d}$, find $y \in \mathbb{W}_{2}(\Omega)$ such that

$$
\langle A(u, y), \varphi\rangle_{\left(\mathbb{W}_{2}(\Omega)\right)^{*} ; \mathbb{W}_{2}(\Omega)}=\langle f, \varphi\rangle_{\left(\mathbb{W}_{2}(\Omega)\right)^{*} ; \mathbb{W}_{2}(\Omega)}, \forall \varphi \in \mathbb{W}_{2}(\Omega)
$$

for which $A(u, y)=f$ is its operator form, has a unique solution $y=y(u) \in$ $\mathbb{W}_{2}(\Omega)$. We note that the duality pairing in the right hand side of (3.12) makes a sense for any distribution $f \in H^{-2}\left(\Omega ; \Gamma_{D}\right)$ because

$$
H^{-2}\left(\Omega ; \Gamma_{D}\right):=\left(W_{0}^{2,2}\left(\Omega ; \Gamma_{D}\right)\right)^{*} \subset\left(\mathbb{W}_{2}(\Omega)\right)^{*}
$$

It remains to show that the solution $y$ of (3.12) satisfies the Minty relation (3.7). Indeed, in view of the monotonicity of $A$, we have

$$
\begin{aligned}
0 & \leq\langle A(u, v)-A(u, y), v-y\rangle_{\left(\mathbb{W}_{2}(\Omega)\right)^{*} ; \mathbb{W}_{2}(\Omega)} \\
& =\langle A(u, v), v-y\rangle_{\left(\mathbb{W}_{2}(\Omega)\right)^{*} ; \mathbb{W}_{2}(\Omega)}-\langle A(u, y), v-y\rangle_{\left(\mathbb{W}_{2}(\Omega)\right)^{*} ; \mathbb{W}_{2}(\Omega)} \\
& \text { by } \stackrel{(3.12)}{=}\langle A(u, v), v-y\rangle_{\left(\mathbb{W}_{2}(\Omega)\right)^{*} ; \mathbb{W}_{2}(\Omega)}-\langle f, \varphi\rangle_{\left(\mathbb{W}_{2}(\Omega)\right)^{*} ; \mathbb{W}_{2}(\Omega)} .
\end{aligned}
$$

Thus,

$$
\langle A(u, v), v-y\rangle_{\left(\mathbb{W}_{2}(\Omega)\right)^{*} ; \mathbb{W}_{2}(\Omega)} \geq\langle f, \varphi\rangle_{H^{-2}\left(\Omega ; \Gamma_{D}\right) ; H_{0}^{2}\left(\Omega ; \Gamma_{D}\right)}, \quad \forall v \in \mathbb{W}_{2}(\Omega),
$$

and, hence, in view of Remark 3.1, the Minty relation (3.7) holds true.

Taking this fact into account, we adopt the following notion. 
Definition 3.2. We say that $(u, y)$ is an admissible pair to the OCP (3.6) if $u \in \mathfrak{A}_{a d} \subset L^{1}(\Omega), y \in \mathbb{W}_{2}(\Omega)$, the pair $(u, y)$ is related by the Minty inequality (3.7), $I(u, y)<+\infty$, and

$$
\gamma_{1}(y) \in L_{+}^{2}\left(\Gamma_{S}\right), \quad \zeta^{\max }-\gamma_{1}(y) \in L_{+}^{2}\left(\Gamma_{S}\right),
$$

where $L_{+}^{2}\left(\Gamma_{S}\right)$ stands for the natural ordering cone of positive elements in $L^{2}\left(\Gamma_{S}\right)$, i.e.

$$
L_{+}^{2}\left(\Gamma_{S}\right):=\left\{v \in L^{2}\left(\Gamma_{S}\right) \mid v \geq 0 \quad \mathcal{H}^{N-1} \text {-a.e. on } \Gamma_{S}\right\} .
$$

We denote by $\Xi$ the set of all admissible pairs for the OCP (3.6). Let $\tau$ be the topology on the set $\Xi \subset L^{1}(\Omega) \times \mathbb{W}_{2}(\Omega)$ which we define as the product of the norm topology of $L^{1}(\Omega)$ and the weak topology of $H_{0}^{2}\left(\Omega ; \Gamma_{D}\right)$. We say that a pair $\left(u^{0}, y^{0}\right) \in L^{1}(\Omega) \times \mathbb{W}_{2}(\Omega)$ is an optimal solution to problem (3.6) if

$$
\left(u^{0}, y^{0}\right) \in \Xi \quad \text { and } \quad I\left(u^{0}, y^{0}\right)=\inf _{(u, y) \in \Xi} I(u, y) .
$$

Remark 3.2. Before we proceed further, we need to make sure that minimization problem (3.6) is meaningful, i.e. there exists at least one pair $(u, y)$ such that $(u, y)$ satisfying the control and state constraints $(3.3)-(3.5), I(u, y)<+\infty$, and $(u, y)$ would be a physically relevant solution to the boundary value problem (3.2)(3.3)? In fact, one needs the set of admissible solutions to be nonempty. But even if we are aware that $\Xi \neq \emptyset$, this set must be sufficiently rich in some sense, otherwise the OCP (3.6) becomes trivial. From a mathematical point of view, to deal directly with the control and especially state constraints is typically very difficult $[5,12,18]$. Thus, the regularity of OCPs with control and state constraints is an open question even for the simplest situation.

It is reasonably now to make use of the following Hypothesis.

$\left(H_{1}\right)$ OCP $(3.6)$ is regular in the following sense - there exists at least one pair $(u, y) \in L^{1}(\Omega) \times \mathbb{W}_{2}(\Omega)$ such that $(u, y) \in \Xi$.

\section{Existence of Optimal Solutions}

In this section we focus on the solvability of optimal control problem (3.2)(3.6). Hereinafter, we suppose that the space $L^{1}(\Omega) \times \mathbb{W}_{p}(\Omega)$ is endowed with the norm $\|(u, y)\|_{L^{1}(\Omega) \times \mathbb{W}_{2}(\Omega)}:=\|u\|_{L^{1}(\Omega)}+\|y\|_{2, \Delta}$.

We begin with a couple of auxiliary results.

Lemma 4.1. Let $\left\{\left(u_{k}, y_{k}\right) \in \Xi\right\}_{k \in \mathbb{N}}$ be a sequence such that $\left(u_{k}, y_{k}\right) \stackrel{\tau}{\longrightarrow}(u, y)$ in $L^{1}(\Omega) \times \mathbb{W}_{2}(\Omega)$. Then we have

$$
\lim _{k \rightarrow \infty} \int_{\Omega} u_{k} \Delta y_{k} \Delta \varphi d x=\int_{\Omega} u \Delta y \Delta \varphi d x \quad \forall \varphi \in C_{0}^{\infty}\left(\Omega ; \Gamma_{D}\right) .
$$


Proof. Since $u_{k} \rightarrow u$ in $L^{1}(\Omega)$ and $\left\{u_{k}\right\}_{k \in \mathbb{N}}$ is bounded in $L^{\infty}(\Omega)$, we get that $u_{k} \rightarrow u$ strongly in $L^{r}(\Omega)$ for every $1 \leq r<+\infty$. In particular, we have that $u_{k} \rightarrow u$ in $L^{2}(\Omega)$ and $\Delta y_{k} \Delta \varphi \rightarrow \Delta y \Delta \varphi$ in $L^{2}(\Omega)$. Hence, it is immediate to pass to the limit and to deduce (4.1).

As a consequence, we have the following property.

Corollary 4.1. Let $\left\{\left(u_{k}, y_{k}\right) \in \Xi\right\}_{k \in \mathbb{N}}$ and $\left\{\zeta_{k} \in H_{0}^{2}\left(\Omega ; \Gamma_{D}\right)\right\}_{k \in \mathbb{N}}$ be sequences such that $\left(u_{k}, y_{k}\right) \stackrel{\tau}{\longrightarrow}(u, y)$ in $L^{1}(\Omega) \times \mathbb{W}_{2}(\Omega)$ and $\zeta_{k} \rightarrow \zeta$ in $H_{0}^{2}\left(\Omega ; \Gamma_{D}\right)$. Then

$$
\lim _{k \rightarrow \infty} \int_{\Omega} u_{k} \Delta y_{k} \Delta \zeta_{k} d x=\int_{\Omega} u \Delta y \Delta \zeta d x
$$

Our next step concerns the study of topological properties of the set of admissible solutions $\Xi$ to problem (3.6).

The following result is crucial for our further analysis.

Theorem 4.1. Let $\left\{\left(u_{k}, y_{k}\right)\right\}_{k \in \mathbb{N}} \subset \Xi$ be a bounded sequence in $B V(\Omega) \times \mathbb{W}_{2}(\Omega)$. Then there is a pair $(u, y) \in L^{1}(\Omega) \times \mathbb{W}_{2}(\Omega)$ such that, up to a subsequence, $\left(u_{k}, y_{k}\right) \stackrel{\tau}{\longrightarrow}(u, y)$ and $(u, y) \in \Xi$.

Proof. By Theorem 2.1 and compactness properties of the space $\mathbb{W}_{2}(\Omega)$, there exists a subsequence of $\left\{\left(u_{k}, y_{k}\right) \in \Xi\right\}_{k \in \mathbb{N}}$, still denoted by the same indices, and functions $u \in B V(\Omega)$ and $y \in \mathbb{W}_{2}(\Omega)$ such that

$$
u_{k} \rightarrow u \text { in } L^{1}(\Omega), \quad y_{k} \rightarrow y \text { in } H_{0}^{2}\left(\Omega ; \Gamma_{D}\right), \text { and, hence, } y_{k} \rightarrow y \text { in } H_{0}^{1}(\Omega) .
$$

Then by Lemma 4.1, we have

$$
\lim _{k \rightarrow \infty} \int_{\Omega} u_{k} \Delta \varphi \Delta y_{k} d x=\int_{\Omega} u \Delta \varphi \Delta y d x, \quad \forall \varphi \in C_{0}^{\infty}\left(\Omega ; \Gamma_{D}\right) .
$$

It remains to show that the limit pair $(u, y)$ is related by inequality $(3.7)$ and satisfies the state constraints (3.13). With that in mind we write down the Minty relation for $\left(u_{k}, y_{k}\right)$ :

$$
\int_{\Omega} u_{k} \Delta \varphi\left(\Delta \varphi-\Delta y_{k}\right) d x \geq\left\langle f, \varphi-y_{k}\right\rangle_{H^{-2}\left(\Omega ; \Gamma_{D}\right) ; H_{0}^{2}\left(\Omega ; \Gamma_{D}\right)}, \quad \forall \varphi \in C_{0}^{\infty}\left(\Omega ; \Gamma_{D}\right) .
$$

In view of (4.2) and Lemma 4.1, we have

$$
\lim _{k \rightarrow \infty} \int_{\Omega}|\Delta \varphi|^{2} u_{k} d x=\int_{\Omega}|\Delta \varphi|^{2} u d x, \quad \lim _{k \rightarrow \infty} \int_{\Omega} u_{k} \Delta \varphi \Delta y_{k} d x=\int_{\Omega} u \Delta \varphi \Delta y d x .
$$

We, thus, can pass to the limit in relation (4.3) as $k \rightarrow \infty$ and arrive at the inequality (3.7), which means that $y \in \mathbb{W}_{2}(\Omega)$ is a weak solution to the boundary value problem (3.2)-(3.3) in the sense of Minty. Since the injections (2.1) are 
compact and the cone $L_{+}^{2}\left(\Gamma_{S}\right)$ is closed with respect to the strong convergence in $L^{2}\left(\Gamma_{S}\right)$, it follows that $\frac{\partial y_{k}}{\partial \nu} \rightarrow \frac{\partial y}{\partial \nu}$ strongly in $L^{2}\left(\Gamma_{S}\right)$ and, hence,

$$
\lim _{k \rightarrow \infty} \gamma_{1}\left(y_{k}\right)=\gamma_{1}(y) \in L_{+}^{2}\left(\Gamma_{S}\right) \text { and } \gamma_{1}(y) \in \zeta^{\max }-L_{+}^{2}\left(\Gamma_{S}\right) .
$$

This fact together with $u \in \mathfrak{A}_{a d}$ leads us to the conclusion: $(u, y) \in \Xi$, i.e. the limit pair $(u, y)$ is admissible to optimal control problem (3.6). The proof is complete.

Remark 4.1. Having applied the arguments of Remark 3.1, it is easy to show that in this case the energy equality (3.9) holds true for every $\tau$-cluster pair $(u, y)$ mentioned in Theorem 4.1.

In conclusion of this section, we give the existence result for optimal pairs to problem (3.6).

Theorem 4.2. Assume that, for given distributions $f \in W^{-2, q}\left(\Omega ; \Gamma_{D}\right), y_{d} \in$ $L^{2}(\Omega)$, and $\zeta^{\text {max }} \in L^{p}(\partial \Omega)$, the Hypothesis $\left(H_{1}\right)$ is valid. Then optimal control problem (3.6) admits at least one solution $\left(u^{\text {opt }}, y^{\text {opt }}\right) \in B V(\Omega) \times \mathbb{W}_{p}(\Omega)$.

Proof. Since the set of admissible pairs $\Xi$ is nonempty and the cost functional is bounded from below on $\Xi$, it follows that there exists a minimizing sequence $\left\{\left(u_{k}, y_{k}\right) \in \Xi\right\}_{k \in \mathbb{N}}$ to problem (3.6). Then the inequality

$$
\inf _{(u, y) \in \Xi} I(u, y)=\lim _{k \rightarrow \infty}\left[\int_{\Omega}\left|y_{k}(x)-y_{d}(x)\right|^{2} d x+\int_{\Omega}\left|D u_{k}\right|\right]<+\infty,
$$

implies the existence of a constant $C>0$ such that

$$
\sup _{k \in \mathbb{N}} \int_{\Omega}\left|D u_{k}\right| \leq C .
$$

Hence, in view of the definition of the class of admissible controls $\mathfrak{A}_{a d}$ and a priori estimate (3.10), the sequence $\left\{\left(u_{k}, y_{k}\right) \in \Xi\right\}_{k \in \mathbb{N}}$ is bounded in $B V(\Omega) \times \mathbb{W}_{p}(\Omega)$. Therefore, by Theorem 4.1, there exist functions $u^{*} \in \mathfrak{A}_{a d}$ and $y^{*} \in \mathbb{W}_{p}(\Omega)$ such that $\left(u^{*}, y^{*}\right) \in \Xi$ and, up to a subsequence, $u_{k} \rightarrow u^{*}$ strongly in $L^{1}(\Omega)$ and $y_{k} \rightarrow y^{*}$ weakly in $W_{0}^{2, p}\left(\Omega ; \Gamma_{D}\right)$. To conclude the proof, it is enough to show that the cost functional $I$ is lower semicontinuous with respect to the tau-convergence. Since $y_{k} \rightarrow y^{*}$ strongly in $L^{p}(\Omega)$ by Sobolev embedding theorem, it follows that

$$
\begin{aligned}
\lim _{k \rightarrow \infty} \int_{\Omega}\left|y_{k}(x)-y_{d}(x)\right|^{2} d x & =\int_{\Omega}\left|y^{*}(x)-y_{d}(x)\right|^{2} d x, \\
\liminf _{k \rightarrow \infty} \int_{\Omega}\left|D u_{k}\right| & \geq \int_{\Omega}\left|D u^{*}\right| \text { by }(2.5) .
\end{aligned}
$$

Thus,

$$
I\left(u^{*}, y^{*}\right) \leq \liminf _{k \rightarrow \infty} I\left(u_{k}, y_{k}\right)=\inf _{(u, y) \in \Xi} I(u, y) .
$$

Hence, $\left(u^{*}, y^{*}\right)$ is an optimal pair, and we arrive at the required conclusion. 


\section{Variational Inequality Approach to Regularization of OCP}

As follows from Theorem 4.2, the existence of optimal solutions to the problem (3.6) can be obtained by using compactness arguments and the regularity assumption $\left(H_{1}\right)$. However, because of the state constraints (3.4) the regularity of the OCP (3.6) is an open question even for the simplest situation. Nevertheless, in many applications it is an important task to find an admissible (or at least an approximately admissible, in a sense to be made precise) solution when both control and state constraints for the OCP are given. Thus, if the set of admissible solutions is rather "thin", it is reasonable to weaken the requirements on admissible solutions to the original OCP. In particular, it would be reasonable to assume that we may satisfy the state equation

$$
\langle A(u, y), \varphi\rangle_{\left(\mathbb{W}_{2}(\Omega)\right)^{*} ; \mathbb{W}_{2}(\Omega)}=\langle f, \varphi\rangle_{\left(\mathbb{W}_{2}(\Omega)\right)^{*} ; \mathbb{W}_{2}(\Omega)}, \forall \varphi \in \mathbb{W}_{2}(\Omega)
$$

and the corresponding state constraint

$$
y \in K:=\left\{v \in \mathbb{W}_{2}(\Omega) \mid \gamma_{1}(v) \in L_{+}^{2}\left(\Gamma_{S}\right), \zeta^{\max }-\gamma_{1}(v) \in L_{+}^{2}\left(\Gamma_{S}\right)\right\}
$$

with some accuracy. Here, the operator $A(\cdot, \cdot): L^{1}(\Omega) \times \mathbb{W}_{2}(\Omega) \rightarrow\left(\mathbb{W}_{2}(\Omega)\right)^{*}$ is defined by the left-hand side of relation (3.11). For this purpose, we make use the following observation: If a pair $(u, y)$ is admissible to the original problem, i.e. $(u, y) \in \Xi$, then this pair satisfies the relation

$$
\langle A(u, y), \zeta-y\rangle_{\left(\mathbb{W}_{2}(\Omega)\right)^{*} ; \mathbb{W}_{p}(\Omega)} \geq\langle f, \zeta-y\rangle_{\left(\mathbb{W}_{2}(\Omega)\right)^{*} ; \mathbb{W}_{p}(\Omega)}, \quad \forall \zeta \in K
$$

for each $\varepsilon>0$.

Note that the reverse statement is not true in general. In fact, we discuss a variant of the penalization approach, called the "variational inequality (VI) method". This idea was first studied in [16]. Thus, if a pair $(u, y) \in \mathfrak{A}_{a d} \times K$ is related by variational inequality (5.1), then it is not necessary to suppose that $(u, y)$ satisfy the operator equation $A(u, y)=f$. In view of this, we can use the penalized term $\|A(u, y)-f\|_{\left(\mathbb{W}_{p}(\Omega)\right)^{*}}$ as a deviation measure in an associated cost functional. As a result, we arrive at the following penalized OCP:

$$
\begin{aligned}
& \text { Minimize }\left\{\widehat{I}_{\varepsilon}(u, y)=\int_{\Omega}\left|y-y_{d}\right|^{2} d x+\int_{\Omega}|D u|+\frac{1}{\varepsilon}\|A(u, y)-f\|_{\left(\mathbb{W}_{2}(\Omega)\right)^{*}}\right\} \\
& \text { subject to the constraints } \\
& \left.\begin{array}{c}
u \in \mathfrak{A}_{a d}, \quad y \in K \\
\langle A(u, y), \zeta-y\rangle_{\left(\mathbb{W}_{2}(\Omega)\right)^{*} ; \mathbb{W}_{2}(\Omega)} \geq\langle f, \zeta-y\rangle_{\left(\mathbb{W}_{2}(\Omega)\right)^{*} ; \mathbb{W}_{2}(\Omega)}, \forall \zeta \in K,
\end{array}\right\} .
\end{aligned}
$$

In a more compact form this problem can be stated as follows

$$
\inf _{(u, y) \in \widehat{\Xi}_{\varepsilon}} \widehat{I}_{\varepsilon}(u, y), \quad \forall \varepsilon>0
$$


where the set of admissible solutions $\widehat{\Xi}_{\varepsilon} \subset L^{1}(\Omega) \times \mathbb{W}_{2}(\Omega)$ we describe as follows: $\widehat{\Xi}_{\varepsilon}:=\left\{(u, y): u \in \mathfrak{A}_{a d}, y \in K, \widehat{I}_{\varepsilon}(u, y)<+\infty\right.$, and $(u, y)$ satisfies VI (5.1) $\}$.

Our aims in this section is to show that penalized OCP (5.4) is solvable for each $\varepsilon>0$ without any assuption about its regularity, and to study the asymptotic properties of sequences of optimal pairs $\left\{\left(u_{\varepsilon}^{0}, y_{\varepsilon}^{0}\right\}_{\varepsilon>0}\right.$ to problem (5.4) when the small parameter $\varepsilon>0$ varies in a strictly decreasing sequence of positive numbers converging to zero. We begin with the following result.

Lemma 5.1. Under assumption (3.1), for every fixed $u \in U_{\partial}$ and $\varepsilon>0$, the variational inequality (5.1) admits at leas one solution $y=y(u)$ such that $y \in K$.

Proof. Let $\varepsilon>0$ be a fixed value. As follows from definition of the set $K, K$ is a nonempty convex closed subset of $\mathbb{W}_{2}(\Omega)$ with respect to the $\|\cdot\|_{2, \Delta}$-norm topology. Due to the assumption (3.1), we have the following estimates

$$
\begin{aligned}
\sup _{\|y\|_{2, \Delta} \leq \rho}\|A(u, y)\|_{\left(\mathbb{W}_{2}(\Omega)\right)^{*}} & =\sup _{\|y\|_{2, \Delta} \leq \rho\|v\|_{2, \Delta} \leq 1} \sup _{\| A(u, y), v\rangle_{\left(\mathbb{W}_{2}(\Omega)\right)^{*} ; \mathbb{W}_{2}(\Omega)}}\langle A \| \\
& =\sup _{\|y\|_{2, \Delta} \leq \rho\|v\|_{2, \Delta} \leq 1} \sup _{\Omega}\left[\int_{\Omega} u y \Delta v d x\right] \\
& \leq \sup _{\|y\|_{2, \Delta} \leq \rho\left\|_{v}\right\|_{2, \Delta} \leq 1}\left[\left\|\xi_{2}\right\|_{L^{\infty}(\Omega)}\|y\|_{2, \Delta}\|v\|_{2, \Delta}\right] \\
& \leq \sup _{\|y\|_{2, \Delta} \leq \rho\|v\|_{2, \Delta} \leq 1}\left[\left\|\xi_{2}\right\|_{L^{\infty}(\Omega)}\|y\|_{2, \Delta}\|v\|_{2, \Delta}\right] \\
& \leq\left\|\xi_{2}\right\|_{L^{\infty}(\Omega)} \rho<+\infty \\
\langle A(u, y), y\rangle_{\left(\mathbb{W}_{2}(\Omega)\right)^{*} ; \mathbb{W}_{2}(\Omega)} & \geq \int_{\Omega} u|\Delta y|^{2} d x \geq \alpha\|y\|_{2, \Delta}^{2} .
\end{aligned}
$$

Hence, for every fixed $u \in \mathfrak{A}_{a d}$, the operator $A(u, \cdot): \mathbb{W}_{2}(\Omega) \rightarrow\left(\mathbb{W}_{2}(\Omega)\right)^{*}$ is bounded and coercive. Moreover, it is shown in [17, Proposition 2.42] that the property (3.1) ensure the following implication

$$
\begin{aligned}
& \left.\begin{array}{c}
y_{k} \rightarrow y \text { in } H_{0}^{2}\left(\Omega ; \Gamma_{D}\right), \\
\limsup _{k \rightarrow \infty}\left\langle A\left(u, y_{k}\right), y_{k}-y\right\rangle_{\left(\mathbb{W}_{2}(\Omega)\right)^{*} ; \mathbb{W}_{2}(\Omega)} \leq 0
\end{array}\right\} \Longrightarrow \\
& \Longrightarrow\left\{\begin{array}{c}
\forall v \in H_{0}^{2}\left(\Omega ; \Gamma_{D}\right) \\
\langle A(u, y), y-v\rangle_{\left(\mathbb{W}_{2}(\Omega)\right)^{*} ; \mathbb{W}_{2}(\Omega)} \leq \liminf _{k \rightarrow \infty}\left\langle A\left(u, y_{k}\right), y_{k}-v\right\rangle_{\left(\mathbb{W}_{2}(\Omega)\right)^{*} ; \mathbb{W}_{2}(\Omega)} .
\end{array}\right.
\end{aligned}
$$

Thus, the operator $A(u, \cdot): \mathbb{W}_{2}(\Omega) \rightarrow\left(\mathbb{W}_{2}(\Omega)\right)^{*}$ is pseudo-monotone for each $u \in \mathfrak{A}_{a d}$. Hence, following the well-know existence result (see, for instance, [3,7]), there exists at least one solution $y=y(u)$ of variational inequality (5.1) such that $y \in K$.

As an obvious consequence of Lemma 5.1, we have the following noteworthy property of penalized OCP (5.2)-(5.3). 
Corollary 5.1. For each $\varepsilon>0$ the set $\widehat{\Xi}_{\varepsilon}$ is nonempty, i.e. the problem (5.2)(5.3) is regular.

To proceed further, we introduce the following notion.

Definition 5.1. An operator $A: \mathfrak{A}_{a d} \times \mathbb{W}_{2}(\Omega) \rightarrow\left(\mathbb{W}_{2}(\Omega)\right)^{*}$ is said to be quasi-monotone if for any sequence $\left\{\left(u_{k}, y_{k}\right)\right\}_{k=1}^{\infty}$ such that $\left\{u_{k}\right\}_{k \in \mathbb{N}} \subset \mathfrak{A}_{a d}$ and $\left(u_{k}, y_{k}\right) \stackrel{\tau}{\rightarrow}(u, y)$ in $L^{1}(\Omega) \times H_{0}^{2}\left(\Omega ; \Gamma_{D}\right)$, the condition

$$
\limsup _{k \rightarrow \infty}\left\langle A\left(u_{k}, y_{k}\right), y_{k}-y\right\rangle_{\left(\mathbb{W}_{2}(\Omega)\right)^{*} ; \mathbb{W}_{2}(\Omega)} \leq 0
$$

implies the relation

$$
\langle A(u, y), y-v\rangle_{\left(\mathbb{W}_{2}(\Omega)\right)^{*} ; \mathbb{W}_{2}(\Omega)} \leq \liminf _{k \rightarrow \infty}\left\langle A\left(u_{k}, y_{k}\right), y_{k}-v\right\rangle_{\left(\mathbb{W}_{2}(\Omega)\right)^{*} ; \mathbb{W}_{2}(\Omega)}
$$

for all $v \in H_{0}^{2}\left(\Omega ; \Gamma_{D}\right)$.

Definition 5.2. We say that an operator $A: \mathfrak{A}_{a d} \times \mathbb{W}_{2}(\Omega) \rightarrow\left(\mathbb{W}_{2}(\Omega)\right)^{*}$ possesses the property $(\mathfrak{M})$, if for any sequence $\left\{\left(u_{k}, y_{k}\right)\right\}_{k=1}^{\infty}$ such that $\left\{u_{k}\right\}_{k \in \mathbb{N}} \subset \mathfrak{A}_{a d}$ and $\left(u_{k}, y_{k}\right) \stackrel{\tau}{\rightarrow}(u, y)$ in $L^{1}(\Omega) \times H_{0}^{2}\left(\Omega ; \Gamma_{D}\right)$, the conditions

$$
\begin{gathered}
A\left(u_{k}, y_{k}\right) \rightarrow d \text { in }\left(\mathbb{W}_{2}(\Omega)\right)^{*} \\
\limsup _{k \rightarrow \infty}\left\langle A\left(u_{k}, y_{k}\right), y_{k}\right\rangle_{\left(\mathbb{W}_{2}(\Omega)\right)^{*} ; \mathbb{W}_{2}(\Omega)} \leq\langle d, y\rangle_{\left(\mathbb{W}_{2}(\Omega)\right)^{*} ; \mathbb{W}_{2}(\Omega)}
\end{gathered}
$$

imply the relation $d=A(u, y)$.

Our next intention is to prove the following crucial result.

Theorem 5.1. The operator $A: \mathfrak{A}_{a d} \times \mathbb{W}_{2}(\Omega) \rightarrow\left(\mathbb{W}_{2}(\Omega)\right)^{*}$, given by formula (3.11), is quasi-monotone provided assumption (3.1) holds true.

Proof. Let $\left\{\left(u_{k}, y_{k}\right)\right\}_{k=1}^{\infty}$ be a sequence such that $\left\{u_{k}\right\}_{k \in \mathbb{N}} \subset \mathfrak{A}_{a d}$ and $\left(u_{k}, y_{k}\right) \stackrel{\tau}{\rightarrow}$ $(u, y)$ in $L^{1}(\Omega) \times H_{0}^{2}\left(\Omega ; \Gamma_{D}\right)$. We assume that inequality (5.6) holds true. Our aim is to establish the relation (5.7). With that in mind, we set

$$
\begin{array}{r}
\langle B(u, v, y), w\rangle_{\left(\mathbb{W}_{2}(\Omega)\right)^{*} ; \mathbb{W}_{2}(\Omega)}:=\int_{\Omega} u \Delta y \Delta w d x, \\
\forall u \in \mathfrak{A}_{a d} \text { and } \forall y, v, w \in H_{0}^{2}\left(\Omega ; \Gamma_{D}\right)
\end{array}
$$

and divide our proof onto several steps.

Step 1. We show that, for each $v \in H_{0}^{2}\left(\Omega ; \Gamma_{D}\right)$,

$$
\begin{aligned}
\lim _{k \rightarrow \infty}\left\langle B\left(u_{k}, y_{k}, v\right), y_{k}-y\right\rangle_{\left(\mathbb{W}_{p}(\Omega)\right)^{*} ; \mathbb{W}_{p}(\Omega)} & \\
& :=\lim _{k \rightarrow \infty} \int_{\Omega} u_{k}|\Delta v|^{p-2} \Delta v\left(\Delta y_{k}-\Delta y\right) d x=0 .
\end{aligned}
$$


Indeed, to begin with, we note that $u_{k} \rightarrow u$ in $L^{r}(\Omega)$ for every $1 \leq r<+\infty$, because $u, u_{k} \in L^{\infty}(\Omega)$ for all $k \in \mathbb{N}$ by the initial assumptions. Hence,

$$
\int_{\Omega}|\Delta v|^{2}\left|u_{k}-u\right|^{2} d x \rightarrow 0, \quad \forall v \in H_{0}^{2}\left(\Omega ; \Gamma_{D}\right)
$$

by Lebesgue Dominated Theorem. Since the sequence $\left\{\zeta_{k}:=u_{k} \Delta v\right\}_{k \in \mathbb{N}}$ is bounded in $L^{2}(\Omega)$ and

$$
\left\|\zeta_{k}-u \Delta v\right\|_{L^{2}(\Omega)}^{2}=\int_{\Omega}|\Delta v|^{2}\left|u_{k}-u\right|^{2} d x,
$$

it follows from (5.10) that $u_{k} \Delta v \rightarrow u \Delta v$ strongly in $L^{2}(\Omega)$. Therefore, the lefthand side of (5.9) tends to zero as $k \rightarrow \infty$ as the product of strongly and weakly convergent sequences and we arrive at the desired property (5.9).

Step 2. Let us show that

$$
\begin{aligned}
& \lim _{k \rightarrow \infty}\left\langle B\left(u_{k}, y_{k}, v\right), w\right\rangle_{\left(\mathbb{W}_{2}(\Omega)\right)^{*} ; \mathbb{W}_{2}(\Omega)}=\lim _{k \rightarrow \infty} \int_{\Omega} u_{k} \Delta v \Delta w d x \\
& =\int_{\Omega} u \Delta v \Delta w d x=\langle B(u, y, v), w\rangle_{\left(\mathbb{W}_{2}(\Omega)\right)^{*} ; \mathbb{W}_{2}(\Omega)}, \quad \forall v, w \in H_{0}^{2}\left(\Omega ; \Gamma_{D}\right) .
\end{aligned}
$$

By analogy with the previous step, we note that $u_{k} \rightarrow u$ in $L^{r}(\Omega)$ for every $1 \leq r<+\infty$. In particular, this yields $u_{k} \varphi \rightarrow u \varphi$ strongly in $L^{2}(\Omega) \forall \varphi \in L^{\infty}(\Omega)$. In view of this, we infer

$$
\lim _{k \rightarrow \infty} \int_{\Omega} u_{k} \Delta w \varphi d x=\int_{\Omega} u \Delta w \varphi d x \quad \forall \varphi \in L^{\infty}(\Omega), \forall w \in H_{0}^{2}\left(\Omega ; \Gamma_{D}\right) .
$$

This means that

$$
u_{k} \Delta w \rightarrow u \Delta w \text { in } L^{1}(\Omega) .
$$

But we also have that the sequence $\left\{u_{k} \Delta w\right\}_{k \in \mathbb{N}}$ is bounded in $L^{2}(\Omega)$. Hence, $u_{k} \Delta w \rightarrow u \Delta w$ in $L^{2}(\Omega)$ for each $w \in H_{0}^{2}\left(\Omega ; \Gamma_{D}\right)$. Since $\Delta v \in L^{2}(\Omega)$ for any $v \in H_{0}^{2}\left(\Omega ; \Gamma_{D}\right)$, it follows that

$$
\lim _{k \rightarrow \infty} \int_{\Omega} u_{k} \Delta w \Delta v d x=\int_{\Omega} u \Delta w \Delta v d x
$$

by definition of the weak convergence in $L^{2}(\Omega)$. Thus, the equality (5.11) holds true.

Step 3. This is the final step in our proof. As follows from (5.8), for every element $v \in H_{0}^{2}\left(\Omega ; \Gamma_{D}\right)$ and each index $k \in \mathbb{N}$, we have the estimate

$$
\begin{aligned}
\left\langle B\left(u_{k}, y_{k}, y_{k}\right)\right. & \left.-B\left(u_{k}, y_{k}, v\right), y_{k}-v\right\rangle_{\left(\mathbb{W}_{2}(\Omega)\right)^{*} ; \mathbb{W}_{2}(\Omega)} \\
& :=\int_{\Omega} u_{k}\left(\Delta y_{k}-\Delta v\right)\left(\Delta y_{k}-\Delta v\right) d x \\
& \geq \alpha \int_{\Omega}\left|\Delta y_{k}-\Delta v\right|^{2} d x>0, \quad \forall y_{k} \neq v \text { in } \Omega .
\end{aligned}
$$


Let $v \in H_{0}^{2}\left(\Omega ; \Gamma_{D}\right)$ be a fixed element. We put $y_{\sigma}=(1-\sigma) y+\sigma v$ for all $\sigma \in[0,1]$. Taking into account the monotonicity condition (5.13), we see that

$$
\left\langle B\left(u_{k}, y_{k}, y_{k}\right)-B\left(u_{k}, y_{k}, y_{\sigma}\right), y_{k}-y_{\sigma}\right\rangle_{\left(\mathbb{W}_{2}(\Omega)\right)^{*} ; \mathbb{W}_{2}(\Omega)} \geq 0 \text {. }
$$

Since $A(u, y)=B(u, y, y)$, it follows from (5.14) that

$$
\begin{aligned}
\sigma\left\langle A\left(u_{k}, y_{k}\right), y-v\right\rangle_{\left(\mathbb{W}_{2}(\Omega)\right)^{*} ; \mathbb{W}_{2}(\Omega)} \geq-\left\langle A\left(u_{k}, y_{k}\right), y_{k}-y\right\rangle_{\left(\mathbb{W}_{2}(\Omega)\right)^{*} ; \mathbb{W}_{2}(\Omega)} \\
+\left\langle B\left(u_{k}, y_{k}, y_{\sigma}\right), y_{k}-y\right\rangle_{\left(\mathbb{W}_{2}(\Omega)\right)^{*} ; \mathbb{W}_{2}(\Omega)} \\
+\sigma\left\langle B\left(u_{k}, y_{k}, y_{\sigma}\right), y-v\right\rangle_{\left(\mathbb{W}_{2}(\Omega)\right)^{*} ; \mathbb{W}_{2}(\Omega)}
\end{aligned}
$$

Passing to the limit in (5.15) as $k \rightarrow \infty$, we obtain

$$
\begin{aligned}
\sigma \liminf _{k \rightarrow \infty}\left\langle A\left(u_{k}, y_{k}\right), y\right. & -v\rangle_{\left(\mathbb{W}_{2}(\Omega)\right)^{*} ; \mathbb{W}_{2}(\Omega)} \\
\geq & -\limsup _{k \rightarrow \infty}\left\langle A\left(u_{k}, y_{k}\right), y_{k}-y\right\rangle_{\left(\mathbb{W}_{2}(\Omega)\right)^{*} ; \mathbb{W}_{2}(\Omega)} \\
& +\liminf _{k \rightarrow \infty}\left\langle B\left(u_{k}, y_{k}, y_{\sigma}\right), y_{k}-y\right\rangle_{\left(\mathbb{W}_{2}(\Omega)\right)^{*} ; \mathbb{W}_{2}(\Omega)} \\
& +\sigma \liminf _{k \rightarrow \infty}\left\langle B\left(u_{k}, y_{k}, y_{\sigma}\right), y-v\right\rangle_{\left(\mathbb{W}_{2}(\Omega)\right)^{*} ; \mathbb{W}_{2}(\Omega)}
\end{aligned}
$$

where

$$
\begin{aligned}
\liminf _{k \rightarrow \infty} & \left\langle B\left(u_{k}, y_{k}, y_{\sigma}\right), y_{k}-y\right\rangle_{\left(\mathbb{W}_{2}(\Omega)\right)^{*} ; \mathbb{W}_{2}(\Omega)} \\
= & \lim _{k \rightarrow \infty}\left\langle B\left(u_{k}, y_{k}, y_{\sigma}\right), y_{k}-y\right\rangle_{\left(\mathbb{W}_{2}(\Omega)\right)^{*} ; \mathbb{W}_{2}(\Omega)} \stackrel{\text { by } \stackrel{(5.9)}{=} 0,}{\limsup _{k \rightarrow \infty}}\left\langle A\left(u_{k}, y_{k}\right), y_{k}-y\right\rangle_{\left(\mathbb{W}_{2}(\Omega)\right)^{*} ; \mathbb{W}_{2}(\Omega)} \stackrel{\text { by }}{\geq} 0,
\end{aligned}
$$

and

$$
\begin{aligned}
\lim _{k \rightarrow \infty}\left\langle B\left(u_{k}, y_{k}, y_{\sigma}\right), y-v\right\rangle_{\left(\mathbb{W}_{2}(\Omega)\right)^{*} ;} \mathbb{W}_{2}(\Omega) & \\
& \stackrel{\stackrel{(5.11)}{=}}{=}\left\langle B\left(u, y, y_{\sigma}\right), y-v\right\rangle_{\left(\mathbb{W}_{2}(\Omega)\right)^{*} ; \mathbb{W}_{2}(\Omega)} .
\end{aligned}
$$

Hence, for each $\sigma \in[0,1]$, we have the inequality

$$
\liminf _{k \rightarrow \infty}\left\langle A\left(u_{k}, y_{k}\right), y-v\right\rangle_{\left(\mathbb{W}_{2}(\Omega)\right)^{*} ; \mathbb{W}_{2}(\Omega)} \geq\left\langle B\left(u, y, y_{\sigma}\right), y-v\right\rangle_{\left(\mathbb{W}_{2}(\Omega)\right)^{*} ; \mathbb{W}_{2}(\Omega)} .
$$

Since the convergence $y_{\sigma} \rightarrow y$ is strong in $H_{0}^{2}\left(\Omega ; \Gamma_{D}\right)$, it follows that $\Delta y_{\sigma} \rightarrow \Delta y$ strongly in $L^{2}(\Omega)$, and therefore,

$$
\left\langle B\left(u, y, y_{\sigma}\right), y-v\right\rangle_{\left(\mathbb{W}_{2}(\Omega)\right)^{*} ; \mathbb{W}_{2}(\Omega)} \stackrel{\sigma \rightarrow 0}{\rightarrow} \int_{\Omega} u \Delta y(\Delta y-\Delta v) d x
$$


As a result, we deduce from (5.17) and (5.18) that

$$
\begin{aligned}
& \liminf _{k \rightarrow \infty}\left\langle A\left(u_{k}, y_{k}\right), y_{k}-v\right\rangle_{\left(\mathbb{W}_{2}(\Omega)\right)^{*} ; \mathbb{W}_{2}(\Omega)} \\
& \geq \liminf _{k \rightarrow \infty}\left\langle A\left(u_{k}, y_{k}\right), y_{k}-y\right\rangle_{\left(\mathbb{W}_{2}(\Omega)\right)^{*} ; \mathbb{W}_{2}(\Omega)} \\
& +\liminf _{k \rightarrow \infty}\left\langle A\left(u_{k}, y_{k}\right), y-v\right\rangle_{\left(\mathbb{W}_{2}(\Omega)\right)^{*} ; \mathbb{W}_{2}(\Omega)} \\
& \geq \liminf _{k \rightarrow \infty}\left\langle A\left(u_{k}, y_{k}\right), y_{k}-y\right\rangle_{\left(\mathbb{W}_{2}(\Omega)\right)^{*} ; \mathbb{W}_{2}(\Omega)} \\
& +\langle A(u, y), y-v\rangle_{\left(\mathbb{W}_{2}(\Omega)\right)^{*} ; \mathbb{W}_{2}(\Omega)} \\
& \geq \liminf _{k \rightarrow \infty}\left\langle B\left(u_{k}, y_{k}, y_{k}\right)-B\left(u_{k}, y_{k}, y\right), y_{k}-y\right\rangle_{\left(\mathbb{W}_{2}(\Omega)\right)^{*} ; \mathbb{W}_{2}(\Omega)} \\
& +\liminf _{k \rightarrow \infty}\left\langle B\left(u_{k}, y_{k}, y\right), y_{k}-y\right\rangle_{\left(\mathbb{W}_{2}(\Omega)\right)^{*} ; \mathbb{W}_{2}(\Omega)} \\
& +\langle A(u, y), y-v\rangle_{\left(\mathbb{W}_{2}(\Omega)\right)^{*} ; \mathbb{W}_{2}(\Omega)} \\
& \text { by } \stackrel{(5.14)}{\geq} \liminf _{k \rightarrow \infty}\left\langle B\left(u_{k}, y_{k}, y\right), y_{k}-y\right\rangle_{\left(\mathbb{W}_{2}(\Omega)\right)^{*} ; \mathbb{W}_{2}(\Omega)} \\
& +\langle A(u, y), y-v\rangle_{\left(\mathbb{W}_{2}(\Omega)\right)^{*} ; \mathbb{W}_{2}(\Omega)} \\
& \text { by } \stackrel{(5.9)}{=}\langle A(u, y), y-v\rangle_{\left(\mathbb{W}_{2}(\Omega)\right)^{*} ; \mathbb{W}_{2}(\Omega)},
\end{aligned}
$$

that is, the inequality (5.7) is valid.

Remark 5.1. In fact (see [12, Remark 3.13]), we have the following implication:

" $A$ is quasi-monotone" $\Longrightarrow$ " $A$ possesses the property $(\mathfrak{M}) . "$

Hence, by Theorem 5.1, we can claim that the operator $A: \mathfrak{A}_{a d} \times \mathbb{W}_{2}(\Omega) \rightarrow$ $\left(\mathbb{W}_{2}(\Omega)\right)^{*}$, which is defined by relation $(3.11)$, possesses the property $(\mathfrak{M})$.

We are now in a position to show that the penalized optimal control problem in the coefficient of variational inequality (5.2)-(5.3) is solvable for each value $\varepsilon>0$.

Lemma 5.2. If the assumption (3.1) is valid, then the OCP (5.2)-(5.3) admits at least one solution $\left(u_{\varepsilon}^{0}, y_{\varepsilon}^{0}\right) \in \widehat{\Xi}_{\varepsilon}$ for every fixed $\varepsilon>0$ and any $f \in H^{-2}\left(\Omega ; \Gamma_{D}\right)$, $y_{d} \in L^{2}(\Omega)$, and $\zeta^{\max } \in L^{2}(\partial \Omega)$.

Proof. Let $\left\{\left(u_{k}, y_{k}\right)\right\}_{k=1}^{\infty} \subset U_{\partial} \times K$ be a minimizing sequence to problem (5.2)(5.3). The coerciveness property (5.5) and estimate

$$
\begin{aligned}
\left\langle A\left(u_{k}, y_{k}\right), y_{k}-\zeta\right\rangle_{\left(\mathbb{W}_{2}(\Omega)\right)^{*} ; \mathbb{W}_{2}(\Omega)} & \leq\left\langle f, y_{k}-\zeta\right\rangle_{\left(\mathbb{W}_{2}(\Omega)\right)^{*} ; \mathbb{W}_{2}(\Omega)} \\
& \leq\|f\|_{H^{-2}\left(\Omega ; \Gamma_{D}\right)}\left\|y_{k}-\zeta\right\|_{2, \Delta}
\end{aligned}
$$

immediately imply that the sequence $\left\{y_{k}\right\}_{k=1}^{\infty}$ is bounded in $H_{0}^{2}\left(\Omega ; \Gamma_{D}\right)$. Indeed, 
using the notations $V=\mathbb{W}_{2}(\Omega)$ and $V^{*}=\left(\mathbb{W}_{2}(\Omega)\right)^{*}$, we have

$$
\begin{aligned}
\frac{\langle A y, y-\zeta\rangle_{V^{*} ; V}}{\|y-\zeta\|_{V}} & \geq \frac{\langle A y, y-\zeta\rangle_{V^{*} ; V}}{\|y\|_{V}+\|\zeta\|_{V}} \\
& =\frac{\langle A y, y-\zeta\rangle_{V^{*} ; V}}{\|y\|_{V}} \cdot \frac{1}{1+\frac{\|\zeta\|_{V}}{\|y\|_{V}}} \rightarrow+\infty \text { as }\|y\|_{V} \rightarrow \infty .
\end{aligned}
$$

On the other hand, from (5.20) it follows that

$$
\frac{\langle A y, y-\zeta\rangle_{V^{*} ; V}}{\|y-\zeta\|_{V}} \leq \frac{\langle f, y-\zeta\rangle_{V^{*} ; V}}{\|y-\zeta\|_{V}} \leq\|f\|_{V^{*}}=\|f\|_{H^{-2}\left(\Omega ; \Gamma_{D}\right)}
$$

So, comparing these two chains of relations, we arrive at the existence of a constant $C>0$ such that $C$ is independent of $u \in \mathfrak{A}_{a d}$ and $\|y\|_{V} \leq C$ as far as $y \in K$ is a solution to (5.2).

Since

$$
\sup _{k \in \mathbb{N}} \int_{\Omega}\left|D u_{k}\right| \leq \sup _{k \in \mathbb{N}} \widehat{I}_{\varepsilon}\left(u_{k}, y_{k}\right)<+\infty
$$

and the set $\mathfrak{A}_{a d} \times K$ is sequentially closed with respect to the $\tau$-convergence, we may assume by Theroem 2.1 that there exists a pair $\left(u_{\varepsilon}^{0}, y_{\varepsilon}^{0}\right) \in \mathfrak{A}_{a d} \times K$ such that $\left(u_{k}, y_{k}\right) \stackrel{\tau}{\rightarrow}\left(u_{\varepsilon}^{0}, y_{\varepsilon}^{0}\right)$. Then passing to the limit in

$$
\left\langle A\left(u_{k}, y_{k}\right), \zeta-y_{k}\right\rangle_{\left(\mathbb{W}_{2}(\Omega)\right)^{*} ; \mathbb{W}_{2}(\Omega)} \geq\left\langle f, \zeta-y_{k}\right\rangle_{\left(\mathbb{W}_{2}(\Omega)\right)^{*} ; \mathbb{W}_{2}(\Omega)}
$$

as $k \rightarrow \infty$, we obtain

$$
\limsup _{k \rightarrow \infty}\left\langle A\left(u_{k}, y_{k}\right), y_{k}-\zeta\right\rangle_{\left(\mathbb{W}_{2}(\Omega)\right)^{*} ; \mathbb{W}_{2}(\Omega)} \leq\left\langle f, \zeta-y_{\varepsilon}^{0}\right\rangle_{\left(\mathbb{W}_{2}(\Omega)\right)^{*} ; \mathbb{W}_{2}(\Omega)}, \quad \forall \zeta \in K .
$$

Having put here $\zeta=y_{\varepsilon}^{0} \in K$, we arrive at the inequality

$$
\limsup _{k \rightarrow \infty}\left\langle A\left(u_{k}, y_{k}\right), y_{k}-y_{\varepsilon}^{0}\right\rangle_{\left(\mathbb{W}_{2}(\Omega)\right)^{*} ; \mathbb{W}_{2}(\Omega)} \leq 0 .
$$

Hence,

$$
\begin{aligned}
\liminf _{k \rightarrow \infty}\left\langle A\left(u_{k}, y_{k}\right), y_{k}-\zeta\right\rangle_{\left(\mathbb{W}_{2}(\Omega)\right)^{*} ; \mathbb{W}_{2}(\Omega)} & \\
& \geq\left\langle A\left(u_{\varepsilon}^{0}, y_{\varepsilon}^{0}\right), y_{\varepsilon}^{0}-\zeta\right\rangle_{\left(\mathbb{W}_{2}(\Omega)\right)^{*} ; \mathbb{W}_{2}(\Omega)}, \quad \forall \zeta \in K,
\end{aligned}
$$

by the quasi-monotonicity property of the operator $A$. Combining this inequality with (5.21), we come to the relation

$$
\left\langle A\left(u_{\varepsilon}^{0}, y_{\varepsilon}^{0}\right), \zeta-y_{\varepsilon}^{0}\right\rangle_{\left(\mathbb{W}_{2}(\Omega)\right)^{*} ; \mathbb{W}_{2}(\Omega)} \geq\left\langle f, \zeta-y_{\varepsilon}^{0}\right\rangle_{\left(\mathbb{W}_{2}(\Omega)\right)^{*} ; \mathbb{W}_{2}(\Omega)} \quad \forall \zeta \in K .
$$

Thus, $\left(u_{\varepsilon}^{0}, y_{\varepsilon}^{0}\right) \in \mathfrak{A}_{a d} \times K$ is an admissible pair to the problem (5.2)-(5.3). 
Let us show that $\left(u_{\varepsilon}^{0}, y_{\varepsilon}^{0}\right)$ is an optimal pair to this problem. As follows from (5.20), the sequence $\left\{A\left(u_{k}, y_{k}\right)\right\}_{k \in \mathbb{N}}$ is bounded in $\left(\mathbb{W}_{p}(\Omega)\right)^{*}$. Let $d$ be its weak limit in $\left(\mathbb{W}_{2}(\Omega)\right)^{*}$ as $k \rightarrow \infty$. Then

$$
\begin{aligned}
\limsup _{k \rightarrow \infty} & \left\langle A\left(u_{k}, y_{k}\right), y_{k}\right\rangle_{\left(\mathbb{W}_{2}(\Omega)\right)^{*} ; \mathbb{W}_{2}(\Omega)} \\
& \leq\left\langle f, y_{\varepsilon}^{0}-\zeta\right\rangle_{\left(\mathbb{W}_{2}(\Omega)\right)^{*} ; \mathbb{W}_{2}(\Omega)}+\langle d, \zeta\rangle_{\left(\mathbb{W}_{2}(\Omega)\right)^{*} ; \mathbb{W}_{2}(\Omega)} \\
& =\left\langle d, y_{\varepsilon}^{0}\right\rangle_{\left(\mathbb{W}_{2}(\Omega)\right)^{*} ; \mathbb{W}_{2}(\Omega)}+\left\langle d-f, \zeta-y_{\varepsilon}^{0}\right\rangle_{\left(\mathbb{W}_{2}(\Omega)\right)^{*} ; \mathbb{W}_{2}(\Omega)} \forall \zeta \in K .
\end{aligned}
$$

Substituting $y_{\varepsilon}^{0}$ for $\zeta$ in the last inequality, we get

$$
\limsup _{k \rightarrow \infty}\left\langle A\left(u_{k}, y_{k}\right), y_{k}\right\rangle_{\left(\mathbb{W}_{2}(\Omega)\right)^{*} ; \mathbb{W}_{2}(\Omega)} \leq\left\langle d, y_{\varepsilon}^{0}\right\rangle_{\left(\mathbb{W}_{2}(\Omega)\right)^{*} ; \mathbb{W}_{2}(\Omega)} .
$$

Since the quasi-monotone operator possesses the $(\mathfrak{M})$-property (see Remark 5.1), it follows that $d=A\left(u_{\varepsilon}^{0}, y_{\varepsilon}^{0}\right)$. As a result, using the $\tau$-lower semicontinuity property of the cost functional (5.2), we finally obtain

$$
\begin{aligned}
\inf _{(u, y) \in \widehat{\Xi}_{\varepsilon}} \widehat{I}_{\varepsilon}(u, y) & =\liminf _{k \rightarrow \infty} \widehat{I}_{\varepsilon}\left(u_{k}, y_{k}\right) \\
& \geq I\left(u_{\varepsilon}^{0}, y_{\varepsilon}^{0}\right)+\varepsilon^{-1}\left\|A\left(u_{\varepsilon}^{0}, y_{\varepsilon}^{0}\right)-f\right\|_{\left(\mathbb{W}_{2}(\Omega)\right)^{*}}=\widehat{I}_{\varepsilon}\left(u_{\varepsilon}^{0}, y_{\varepsilon}^{0}\right) .
\end{aligned}
$$

Thus, $\left(u_{\varepsilon}^{0}, y_{\varepsilon}^{0}\right)$ is an optimal pair to the penalized problem (5.2)-(5.3).

The next step of our analysis is to consider a sequence of optimal pairs $\left\{\left(u_{\varepsilon}^{0}, y_{\varepsilon}^{0}\right)\right\}_{\varepsilon>0} \subset \mathfrak{A}_{a d} \times K$ in the limit as $\varepsilon$ tends to 0 .

Theorem 5.2. In addition to the assumptions of Lemma 5.2, assume that the OCP (3.6) is regular, that is the Hypothesis $\left(H_{1}\right)$ is valid. Let $\left\{\left(u_{\varepsilon}^{0}, y_{\varepsilon}^{0}\right)\right\}_{\varepsilon>0}$ be a sequence of optimal pairs to penalized problems (5.2)-(5.3). Then this sequence is relatively compact with respect to the $\tau$-convergence and each of its $\tau$-cluster pair $\left(u^{0}, y^{0}\right)$ is such that (up to a subsequence)

$$
\begin{gathered}
\left(u_{\varepsilon}^{0}, y_{\varepsilon}^{0}\right) \stackrel{\tau}{\longrightarrow}\left(u^{0}, y^{0}\right) \quad \text { as } \varepsilon \rightarrow 0 \\
\left(u^{0}, y^{0}\right) \in \Xi, \quad \text { and } \quad I\left(u^{0}, y^{0}\right)=\inf _{(u, y) \in \Xi} I(u, y) .
\end{gathered}
$$

Proof. Let $\left\{\left(u_{\varepsilon}^{0}, y_{\varepsilon}^{0}\right)\right\}_{\varepsilon>0}$ be a given sequence of optimal pairs to penalized problems (5.2)-(5.3). Since the set $K \subset W_{0}^{2, p}\left(\Omega ; \Gamma_{D}\right)$ contains zero, we have

$$
\begin{aligned}
\alpha\|y\|_{H_{0}^{2}\left(\Omega ; \Gamma_{D}\right)}^{2} & \leq \int_{\Omega} u_{\varepsilon}^{0}\left|\Delta y_{\varepsilon}^{0}\right|^{2} d x=\left\langle A\left(u_{\varepsilon}^{0}, y_{\varepsilon}^{0}\right), y_{\varepsilon}^{0}\right\rangle_{\left(\mathbb{W}_{2}(\Omega)\right)^{*} ; \mathbb{W}_{2}(\Omega)} \\
& \stackrel{\text { by }}{\leq}\langle f .1) \\
\leq & \left\langle f, y_{\varepsilon}^{0}\right\rangle_{\left(\mathbb{W}_{2}(\Omega)\right)^{*} ; \mathbb{W}_{2}(\Omega)} \leq\|f\|_{H^{-2}\left(\Omega ; \Gamma_{D}\right)}\left\|y_{\varepsilon}^{0}\right\|_{2, \Delta} .
\end{aligned}
$$

Hence, the following estimate for the optimal states takes place

$$
\left\|y_{\varepsilon}^{0}\right\|_{2, \Delta} \leq\left(\alpha^{-1}\|f\|_{H^{-2}\left(\Omega ; \Gamma_{D}\right)}\right), \quad \forall u \in \mathfrak{A}_{a d} .
$$


Let us show that the sequence of corresponding optimal controls $\left\{u_{\varepsilon}^{0}\right\}_{\varepsilon>0}$ is $B V$ bounded. Indeed, let $(u, y) \in \Xi$ be any admissible pair to the original OCP (3.6). Then $\widehat{I}_{\varepsilon}(u, y)=I(u, y)$ for each $\varepsilon>0$. On the other hand, since $\left(u_{\varepsilon}^{0}, y_{\varepsilon}^{0}\right)$ is an optimal pair to problem $(5.2)-(5.3)$, this yields $\widehat{I}_{\varepsilon}\left(u_{\varepsilon}^{0}, y_{\varepsilon}^{0}\right) \leq \widehat{I}_{\varepsilon}(u, y)=I(u, y)$ for every $\varepsilon>0$. So, the numerical sequence $\left\{\widehat{I}_{\varepsilon}\left(u_{\varepsilon}^{0}, y_{\varepsilon}^{0}\right)\right\}_{\varepsilon>0}$ is uniformly bounded with respect to $\varepsilon$. Hence, in view of the structure of the cost functional (5.2), we deduce

$$
\left\|A\left(u_{\varepsilon}^{0}, y_{\varepsilon}^{0}\right)-f\right\|_{\mathbb{Y}^{*}} \leq \varepsilon I(u, y), \quad \int_{\Omega}\left|D u_{\varepsilon}^{0}\right| \leq I(u, y) .
$$

From this, we immediately conclude that $\sup _{\varepsilon>0}\left\|u_{\varepsilon}^{0}\right\|_{B V(\Omega)}<+\infty$, and, hence, due to Theorem 2.1 and estimate (5.24), we may assume that there exists a pair $\left(u^{0}, y^{0}\right) \in \mathfrak{A}_{a d} \times K$ such that $\left(u_{\varepsilon}^{0}, y_{\varepsilon}^{0}\right) \stackrel{\tau}{\rightarrow}\left(u^{0}, y^{0}\right)$ as $\varepsilon \rightarrow 0$ in $L^{1}(\Omega) \times H_{0}^{2}\left(\Omega ; \Gamma_{D}\right)$.

Let us show that the pair $\left(u^{0}, y^{0}\right)$ is admissible to the original problem (3.6). Using the arguments of the proof of Lemma 5.2, we have $A\left(u_{\varepsilon}^{0}, y_{\varepsilon}^{0}\right) \rightarrow d$ in $\left(\mathbb{W}_{2}(\Omega)\right)^{*}$ and $d=A\left(u^{0}, y^{0}\right)$. Then, as follows from (5.25), we have

$$
\begin{aligned}
0 \leq\left\|A\left(u^{0}, y^{0}\right)-f\right\|_{\left(\mathbb{W}_{2}(\Omega)\right)^{*}} & \leq \liminf _{\varepsilon \rightarrow 0}\left\|A\left(u_{\varepsilon}^{0}, y_{\varepsilon}^{0}\right)-f\right\|_{\left(\mathbb{W}_{2}(\Omega)\right)^{*}} \\
& =\lim _{\varepsilon \rightarrow 0}\left\|A\left(u_{\varepsilon}^{0}, y_{\varepsilon}^{0}\right)-f\right\|_{\left(\mathbb{W}_{2}(\Omega)\right)^{*}}=0 .
\end{aligned}
$$

Thus, $A\left(u^{0}, y^{0}\right)=f$ as elements of $\left(\mathbb{W}_{2}(\Omega)\right)^{*}$ and, hence, $\left(u^{0}, y^{0}\right) \in \Xi$.

It remains to prove that $\left(u^{0}, y^{0}\right)$ is an optimal pair. If, on the contrary, we assume that the exists a pair $\left(u^{*}, y^{*}\right) \in \Xi$ such that $I\left(u^{*}, y^{*}\right)<I\left(u^{0}, y^{0}\right)$, then

$$
I\left(u_{\varepsilon}^{0}, y_{\varepsilon}^{0}\right) \leq I\left(u_{\varepsilon}^{0}, y_{\varepsilon}^{0}\right)+\varepsilon^{-1}\left\|A\left(u_{\varepsilon}^{0}, y_{\varepsilon}^{0}\right)-f\right\|_{\left(\mathbb{W}_{2}(\Omega)\right)^{*}} \leq I\left(u^{*}, y^{*}\right), \quad \forall \varepsilon>0 .
$$

Therefore, passing to the limit in this inequality as $\varepsilon \rightarrow 0$ and using the $\tau$-lower semicontinuity property of the cost functional, we finally get

$$
I\left(u^{0}, y^{0}\right) \leq \liminf _{\varepsilon \rightarrow 0} \leq I\left(u^{*}, y^{*}\right) .
$$

This contradiction immediately leads us to the conclusion: The $\left(u^{0}, y^{0}\right)$ is optimal the OCP (3.6).

Remark 5.2. As follows from Theorem 5.2, whatever sequence of optimal solutions $\left\{\left(u_{\varepsilon}^{0}, y_{\varepsilon}^{0}\right)\right\}_{\varepsilon>0}$ to the penalized problems (5.2)-(5.3) has been chosen, it always gives in the limit as $\varepsilon \rightarrow 0$ some optimal pair to the original OCP (3.6). However, it is unknown whether the entire set of the solutions to OCP (3.6) can be attained in such way.

\section{References}

1. R. Adams, Sobolev spaces, Academic Press, New York, 1975.

2. H. Attouch, G. Buttazzo, G. Michaille, Variational Analysis in Sobolev and BV Spaces: Application to PDE and Optimization, SIAM, Philadelphia, 2006. 
3. V. Barbu, Analysis and Control of Infinite Dimensional Systems, Academic Press, New York, 1993.

4. D. Bucur, F. Gazzola, The first biharmonic Steklov eiginvalue: positivity preserving and shape optimization, Milan Journal of Mathematics, 79(1)(2011), 247-258.

5. E. Casas, Optimal control in the coefficients of elliptic equations with state constraints, Appl. Math. Optim., 26, 21-37 (1992).

6. F. Colasuonno, P. Pucci, Multiplicity of solutions for $p(x)$-polyharmonic elliptic Kirchoff equations, Nonlinear Analysis, Theory, Methods, and Applications, $\mathbf{7 4}(17)(2011), 5962-5974$.

7. I. Ekeland, R. Temam, Convex Analysis and Variational Problems, North-Holland, Elserver, New York, 1976.

8. F. Gazzola, H.-C. Grunau, G. Sweers, Polyharmonic Boundary Value Problems: Positivity Preserving and Nonlinear Higher Order Elliptic Equations in Bounded Domains, Series: Lecture Notes in Mathematics, Vol.1991, Springer-Verlag, Berlin, 2010 .

9. D. Gilbarg, N.S. Trudinger, Elliptic Partial Differential Equations of Second Order, Springer-Verlag, Berlin, 2001.

10. E. Giusti, Minimal Surfaces and Functions of Bounded Variation, Birkhäuser, Boston, 1984.

11. D. Kinderlehrer, G. Stampacchia, An Introduction to Variational Inequalities and Their Applications, Academic Press, New York, 1980.

12. P. I. Kogut, G. Leugering, Optimal control problems for partial differential equations on reticulated domains. Approximation and Asymptotic Analysis, Series: Systems and Control, Birkhäuser Verlag, Boston, 2011.

13. J.-L. Lions, Some methods of Solving Non-Linear Boundary Value Problems, DunodGauthier-Villars, Paris, 1969.

14. J.-L. Lions, E. Magenes, Problèmes aux Limites non Homogènes et Applications, Vol.1, Travaux et Recherches Mathématiques, No.17, Dunon, Paris, 1968.

15. V.F. Lubyshev, Multiple solutions of an even-order nonlinear problems with convexconcave nonlinearity, Nonlinear Analysis, Theory, Methods, and Applications, 74(4)(2011), 1345-1354.

16. V. S. Mel'nik., Method of monotone operators in the theory of constrained optimal system, Rep. Ukrain. Acad. Sci., A(7)(1986), 64-67.

17. T. Roubicek, Nonlinear Partial Differential Equations with Applications, Birkhäuser, Basel, 2013.

18. T. Roubicek, Relaxation in Optimization Theory and Variational Calculus, De Gruyter series in Nonlinear Analysis and Applications:4, De Gruyter, Berlin, New York, 1997. 